\title{
Travailler au-delà de 48 heures par semaine
}

Working 48 hours and more per Week

\section{François-Xavier Devetter}

\section{(2) OpenEdition}

\section{Journals}

Édition électronique

URL : http://journals.openedition.org/travailemploi/1955

DOI : 10.4000/travailemploi.1955

ISSN : 1775-416X

\section{Éditeur}

DARES - Ministère du Travail

\section{Édition imprimée}

Date de publication : 15 juin 2008

Pagination : 59-70

ISSN : 0224-4365

\section{Référence électronique}

François-Xavier Devetter, «Travailler au-delà de 48 heures par semaine », Travail et Emploi [En ligne], 114 | avril-juin 2008, mis en ligne le 05 novembre 2010, consulté le 08 avril 2021. URL : http:// journals.openedition.org/travailemploi/1955; DOI : https://doi.org/10.4000/travailemploi.1955 


\title{
Travailler au-delà de $\mathbf{4 8}$ heures par semaine
}

\author{
François-Xavier Devetter (*)
}

\begin{abstract}
Malgré les mesures de réduction du temps de travail, les longues durées de travail n'ont pas disparu en France. Les rares recherches menées sur les TLD (très longues durées, atteignant ou dépassant les 48 heures) ne portent que sur quelques groupes de cadres. L'auteur cherche, à partir de l'enquête "Emploi » 2005, à évaluer la situation. Il constate que la population concernée est assez hétérogène et étudie successivement le cas des cadres et professions intermédiaires d'entreprise et celui des employés ou ouvriers peu ou non qualifiés. Les offres de temps de travail très long des employés et ouvriers, par rapport aux logiques qui l'emportent chez les cadres, relèvent d'explications très différentes. Si toutes les catégories socioprofessionnelles sont représentées, quelques professions concentrent l'essentiel des salariés TLD. Les longues durées marquent un marché du travail en France segmenté, où les contraintes liées au temps sont inégalitaires et où les compensations suivent des logiques parfois opposées.
\end{abstract}

Depuis quelques années, le thème de la réduction du temps de travail est en partie écarté au profit du débat sur la «liberté du temps de travail». La question centrale serait ainsi de permettre à chacun de travailler la durée qu'il souhaite notamment «pour gagner plus» sans contrainte légale spécifique. Plusieurs pays anglo-saxons comme l'Australie refusent ainsi toujours d'encadrer le temps de travail, qui demeure un objet de libre négociation entre employeurs et salariés. Depuis un siècle, la France, à l'image de la plupart des pays européens, a choisi une autre voie en fixant des maxima quotidiens et hebdomadaires au nom de la protection du salarié, mais aussi en raison des nombreuses externalités médicales et sociales des durées de travail excessives (Bourdieu, Reynaud, 2004). Cependant au niveau français (par exemple GoDET, 2006) ou européen (avec les débats sur la directive «temps de travail »), la question de la légitimité de la régulation publique (et de l'instauration de durées maximales) est de plus en plus posée.

Malgré la réglementation, les longues durées de travail n'ont pas disparu en France comme en Europe. Pour définir les longues durées de travail, nous avons retenu les durées habituelles de travail telles qu'elles apparaissent dans l'enquête «Emploi » en continu depuis 2003. Parallèlement, parce que les durées annuelles restent relativement déconnectées de la représentation des salariés et qu'au contraire la semaine reste une référence sociale forte, nous avons privilégié les durées hebdomadaires. Enfin nous avons retenu le seuil de 48 heures qui semble

(*) MCF sciences économiques, CLERSE - UMR 8019; devetter@telecom-lille1.eu le plus significatif dans le cas des pays de l'OCDE. En effet, plusieurs textes juridiques s'appuient sur cette limite (droit du travail français pour définir la durée légalement maximale, directive européenne de 1993) tandis que des organismes internationaux comme le BIT recommandent cette durée comme plafond. Cette recommandation s'appuie sur de nombreuses études médicales sur les risques des longues durées (Spurgeon, 2003). Enfin, la durée de 48 heures apparaît comme un seuil statistique important qui «isole» environ $10 \%$ des salariés. En ce sens il s'agit d'un comportement clairement minoritaire qui s'écarte de la «norme temporelle dominante» (DeVETTER, 2002). Nous parlerons donc ici de «très longues durées » (TLD) pour les durées habituelles qui atteignent ou dépassent 48 heures par semaine.

Ces longues durées de travail et les coûts médicaux, sociaux (Pocock, 2001) et environnementaux (SCHOR, 2005) qu'ils impliquent, sont cependant inégalement répartis. Non seulement toutes les catégories sociales ne sont pas touchées uniformément, mais surtout, parmi les professions concernées, la répartition de ces horaires longs et la compensation de leurs contraintes obéissent à des logiques parfois opposées. Les longues durées illustrent ainsi un marché du travail segmenté où les contraintes temporelles sont largement inégalitaires.

Après avoir précisé l'ampleur du phénomène en France en 2005 pour l'ensemble des actifs, puis pour les seuls salariés, nous distinguerons deux groupes principaux de salariés concernés : il s'agit d'un côté des cadres et professions intermédiaires d'entreprises (ce qui nous conduira ainsi a écarter les fonctionnaires) et de l'autre des employés et ouvriers peu ou non qualifiés. 


\section{Quelle est l'ampleur des longues durées en France?}

La fréquence des longues durées est fortement dépendante de la délimitation des catégories étudiées. Nous partirons ainsi d'une vision large incluant l'ensemble des actifs avant de restreindre l'analyse aux seuls salariés au sens de l'enquête «Emploi»(1).

\section{Un phénomène encore important et relativement stable}

Les longs horaires sont une pratique courante dans les pays anglo-saxons où 20 à $30 \%$ de la population active est concernée. Mais comme le rappellent BESCOND et al. (2003), ils ne sont pas pour autant absents des pays méditerranéens (15 à $20 \%$ des actifs) ni même des pays continentaux (aux alentours de $10 \%$ de la population active en Allemagne, en Belgique ou au Danemark). Les longues durées de travail sont ainsi un phénomène qui touche, certes à des degrés divers, tous les pays européens. L'Union européenne s'est ainsi emparée de cette question à l'occasion de la modification de la directive «temps de travail» de 1993, qui fixe la durée maximale hebdomadaire à 48 heures mais maintient de nombreuses possibilités d'opt-out (permettant à une branche, une entreprise, voire un individu de déroger à la règle des 48 heures hebdomadaires maximum), largement utilisées au Royaume-Uni par exemple. La révision de cette directive et notamment la suppression des possibilités de dérogation se heurte à l'opposition des pays les moins régulés et connaissant les durées les plus longues (Royaume-Uni, Pologne notamment). Aucun consensus n'a ainsi pu aboutir lors des derniers conseils européens et la directive est, au premier semestre 2008, toujours bloquée.

Certes la France demeure bien moins concernée que les pays anglo-saxons, mais ce sont néanmoins près de $9 \%$ des salariés et $14 \%$ des actifs occupés qui effectuent de très longues semaines, cette situation étant quasiment stable depuis vingt ans.

Tableau 1 : Proportion d'actifs déclarant des durées hebdomadaires supérieures ou égales à 48 heures

\begin{tabular}{|l|c|l|c|c|}
\hline & $\mathbf{1 9 9 0}$ & $\mathbf{1 9 9 5}$ & $\mathbf{2 0 0 0}$ & $\mathbf{2 0 0 5}$ \\
\hline Agriculteur & 53 & 57 & 61 & 70 \\
\hline Artisan, commerçant... & 53 & 56 & 59 & 62 \\
\hline Cadres & 31 & 31 & 28 & 29 \\
\hline Professions intermédiaires & 12 & 12,5 & 10 & 9 \\
\hline Employés & 6,2 & 6,5 & 5,7 & 5 \\
\hline Ouvriers & 5,5 & 5,5 & 5 & 4 \\
\hline Actifs occupés & 16 & 16 & 14 & 15 \\
\hline Dont salariés & 9,5 & 10 & 9 & 8,5 \\
\hline
\end{tabular}

Pour les années 1990 à 2000, il s'agit des durées réellement accomplies la semaine précédent l'enquête. Pour 2005, il s'agit des durées moyennes dans l'emploi principal (voir note 2).

Sources: enquêtes «Emploi», INSEE.

(1) La seconde partie prolongera ce «resserrement» de l'analyse en écartant les fonctionnaires.
En effet, bien que les données ne soient pas parfaitement comparables (2), le tableau 1 montre que la fréquence de ces durées a tendance à être croissante chez les non salariés, mais qu'elle tend à décroître légèrement, depuis la fin des années 1990, chez les salariés. Sans chercher à expliquer de manière précise cette tendance chez les non salariés, nous pouvons souligner que leurs horaires de travail sont de plus en plus variables (MissÈgue, 2000), ce qui illustre notamment les pressions plus fortes d'une logique marchande qui favorise parallèlement les longues durées de travail. De même la diminution nette du nombre d'aides familiaux parmi les indépendants peut également expliquer partiellement la croissance de la proportion de longs horaires.

Les inégalités de temps de travail se seraient ainsi creusées principalement durant les années 1990, opposant les indépendants et les cadres d'un côté et les autres salariés de l'autre. Il convient cependant d'insister sur la prudence nécessaire dans l'analyse des durées de travail déclarées par les non-salariés et les cadres, ceux-ci ayant des durées moins contrôlées et plus variables. Néanmoins, tout en gardant cette précaution à l'esprit, nous pouvons constater que l'écart de durée hebdomadaire moyenne entre les cadres et les employés est passé de 6 heures à 8 heures 30 entre 1990 et 2005 (soit de $16 \%$ à $25 \%$ de la durée hebdomadaire des employés). Celuici provient essentiellement du développement du travail à temps partiel chez les employés jusqu'en 1998 puis de la mise en place des 35 heures mais également du maintien des longues durées chez les cadres. Ces résultats rejoignent ceux de GershunY (2005) dans le cas de la Grande-Bretagne, où la faiblesse de la régulation (notamment le recours toujours possible à la clause d'opt-out vis-à-vis de la directive «temps de travail» limitant la durée hebdomadaire à 48 heures) favorise un étalement des durées de travail.

(2) Depuis 2003, l'enquête «Emploi» est trimestrielle et sa collecte s'opère en continu alors qu'auparavant elle était établie en mars de chaque année. Plus encore, la mesure de la durée du travail a été modifiée: le concept de durée habituelle a été précisé (pour la distinguer de la durée théorique contractuelle). Ainsi, dans les questionnaires antérieurs à 2002, la distinction entre horaires théoriques et durée effective moyenne n'était pas aussi explicite: «Certains enquêtés déclaraient donc la durée prévue au contrat, d'autres la durée effective moyenne et la durée habituelle moyenne se situait entre les deux» (GIVorD, 2003, p. 61). Les durées effectives à temps complet étant traditionnellement plus longues que les durées théoriques, les enquêtes antérieures à 2002 pouvaient légèrement minorer la proportion de longues durées hebdomadaires. Par ailleurs les individus n'ayant pas de durée habituelle déclarent désormais une durée moyenne fondée sur le mois écoulé avant l'enquête. La qualité de l'évaluation est alors potentiellement moins bonne. Ce problème renvoie à la question plus large de la mesure des durées de travail pour les catégories les moins «contrôlées» comme les cadres et non-salariés. Il convient donc s'insister sur la prudence nécessaire dans l'utilisation des durées de travail déclarées par ces professions, par ailleurs surreprésentées parmi les plus longues durées. 
Ainsi, contrairement aux temps de travail supérieurs à 40 heures (ARNAUDO et al., 2005), on ne peut pas constater la réduction de la fréquence des très longues durées. Bien au contraire, $9 \%$ des salariés effectuent toujours des horaires moyens supérieurs ou égaux à 48 heures en dépit de leur illégalité et de la réduction du temps de travail consécutive aux lois «Aubry».

Malgré ces évolutions, la catégorie des actifs TLD regroupe des situations très diverses. Tant en France qu'à l'étranger, l'attention est souvent portée sur les indépendants et les cadres. Certes ces deux catégories sont proportionnellement bien plus touchées que les autres salariés (voir tableau 1). Pour autant, limiter les TLD aux seuls indépendants et cadres serait très réducteur. En effet si l'on inverse le mode de calcul en regardant non plus la proportion de chaque catégorie concernée mais plutôt la composition par PCS de la population travaillant des TLD, il ressort que les TLD se composent de trois groupes quantitativement comparables au sein des actifs occupés: $39 \%$ sont agriculteurs, commerçants, artisans ou chefs d'entreprises, $30 \%$ sont cadres, et $31 \%$ sont salariés non-cadres.

Cet article visant prioritairement les salariés du secteur privé, nous écarterons tout d'abord le premier groupe. En effet, non seulement la situation des indépendants est mieux connue et moins surprenante, mais surtout leur temps de travail obéit à des logiques sensiblement différentes et demande ainsi une réflexion spécifique (MissèGUe, 2000). La situation des fonctionnaires est aussi particulière, bien qu'ils soient aussi souvent soumis à de longues durées hebdomadaires (ainsi selon l'enquête emploi 2005 près de $10 \%$ des salariés de l'État et $5 \%$ de ceux des collectivités territoriales connaissent des horaires longs) et qu'ils subissent également des contraintes temporelles lourdes (travail de nuit, du week-end ou horaires imprévisibles, voir DeVETTER, 2004 par exemple). L'organisation de leurs temps de travail obéit cependant à des logiques originales (spécificités des enseignants, continuité du service public notamment), c'est pourquoi nous les écarterons également de l'analyse par la suite (voir infra). Les salariés cadres et non cadres du secteur privé sont donc au cœur de cette étude.

\section{Une population assez hétérogène}

Les rares mentions des longues durées de travail en France, mais c'est le cas également de la majorité des recherches menées dans les pays anglo-saxons, ne concernent que des catégories très restreintes de salariés. Les temps de travail supérieurs à 48 heures ne sont étudiés qu'à propos de quelques groupes spécifiques de cadres. Les ingénieurs informatiques apparaissent comme le symbole de ce type de temps de travail au même titre que les caissières de la grande distribution peuvent incarner le temps partiel. Pourtant, la population effectuant de très

\section{Encadré 1 \\ Méthodologie}

Afin de repérer les corrélations les plus fortes en isolant certaines variables pour tenter de raisonner "toutes choses égales par ailleurs", nous avons complété l'analyse par une régression logistique. L'économétrie des variables qualitatives permet en effet d'isoler l'effet d'un seul facteur (l'âge ou le sexe par exemple) sur une variable choisie (le fait de pratiquer de longs horaires). Le modèle utilisé ici est un logit dichotomique appliqué aux données de l'enquête «Emploi » 2005. Les variables explicatives retenues concernent d'une part les caractéristiques personnelles (sexe, âge, situation familiale), et d'autre part celles de l'emploi (profession, employeur, effectifs de l'entreprise). Dans le modèle utilisé ici, la variable expliquée est dichotomique: longs horaires ou absence de longs horaires. Les variables explicatives sont représentées par les modalités des différentes variables, et chaque fois une «modalité de référence" est choisie. Ce choix n'ayant pas d'impact sur les résultats eux-mêmes, nous avons privilégié ceux qui permettaient la meilleure lisibilité. Les différents tests de significativité sont repris dans les tableaux suivants et sont relativement satisfaisants. Le modèle présente un pourcentage de concordance correcte (supérieur à $79 \%$ ). Pour autant il est évident qu'un certain nombre de facteurs sont laissés dans l'ombre et tout particulièrement la durée de travail du conjoint, cet élément étant plus délicat à traiter à partir de l'enquête «Emploi". D'autres variables comme le nombre d'enfants ou le secteur d'activité ont été abandonnées du fait de leur faible significativité. Enfin, des analyses plus spécifiques sur les conditions de travail ont pu bénéficier des enseignements de l'enquête «Conditions de travail " coordonnée par la Dares en complément à l'enquête «Emploi » 2005.

longs horaires hebdomadaires est beaucoup plus diverse et hétérogène que ne le résumerait la seule figure de l'informaticien.

Dans l'optique de présenter une photographie des salariés effectuant de longues durées de travail hebdomadaires il convient dans un premier temps de repérer les caractéristiques les plus marquantes des salariés concernés. Le tableau 2 reprend la proportion de salariés concernés, le poids de chaque groupe au sein des salariés pratiquant des longues durées et fournit également les "odd ratios» issus de l'analyse logistique (voir encadré 1). Il est cependant essentiel de noter qu'il s'agit ici de souligner des corrélations sans présumer immédiatement d'une causalité et encore moins du sens de celle-ci. En effet, il est délicat de tirer des conclusions immédiates sur un éventuel effet causal entre ces caractéristiques et la fréquence des longues durées. Ainsi, par exemple, les hommes peuvent être surreprésentés parmi les TLD d'abord parce qu'ils sont bien plus souvent cadres commerciaux. C'est pourquoi dans un premier temps nous nous limitons à souli- 
gner les grands traits des salariés les plus touchés en insistant sur le fait qu'il s'agit de concomitances: le fait d'être un homme est associé à un plus grand risque de travailler longtemps mais n'explique pas forcément ces comportements. Ce travail permet cependant de décrire la population concernée par les longues durées et de repérer les caractéristiques associées à cette situation.

Tableau 2 : Influence des variables sociodémographiques sur la fréquence des longues durées de travail parmi l'ensemble des salariés (y compris de la fonction publique)

\begin{tabular}{|c|c|c|c|c|}
\hline & & $\begin{array}{l}\text { Influence sur la } \\
\text { fréquence des longues } \\
\text { durées dans le modèle } \\
\text { logistique (odd ratio) }\end{array}$ & $\begin{array}{l}\text { Pourcentage de sala- } \\
\text { riées concernées par les } \\
\text { longues durées }\end{array}$ & $\begin{array}{l}\text { Pourcentage parmi } \\
\text { les salariés effec- } \\
\text { tuant de longues } \\
\text { durées }\end{array}$ \\
\hline \multicolumn{5}{|l|}{ Variables } \\
\hline \multirow[t]{2}{*}{ Sexe } & Femme & Réf. & 5 & 27 \\
\hline & Homme & 3,488 & 12 & 73 \\
\hline \multirow[t]{3}{*}{$\begin{array}{l}\text { Situation du } \\
\text { conjoint }\end{array}$} & Absence de conjoint & Réf. & 6 & 19 \\
\hline & Conjoint actif occupé & 1,361 & 9 & 64 \\
\hline & $\begin{array}{l}\text { Conjoint inactif } \\
\text { ou chômeur }\end{array}$ & 1,460 & 12 & 17 \\
\hline \multirow[t]{5}{*}{ Âge } & $15-29$ & Réf. & 5 & 13 \\
\hline & $30-39$ & 1,337 & 9 & 29 \\
\hline & $40-49$ & 1,388 & 10 & 31 \\
\hline & $50-59$ & 1,366 & 10 & 25 \\
\hline & 60 et plus & $1,207 *$ & 12 & 3 \\
\hline \multicolumn{5}{|l|}{ PCS } \\
\hline & 3 - Cadres & 23,576 & 27 & 47 \\
\hline & $\begin{array}{l}41 \text { - Professions intermédiaires de } \\
\text { l'enseignement, de la santé, de la } \\
\text { fonction publique }\end{array}$ & 4,972 & 5 & 6 \\
\hline & $\begin{array}{l}46 \text { - Professions inter. admi- } \\
\text { nistratives et commerciales des } \\
\text { entreprises }\end{array}$ & 9,883 & 12 & 11 \\
\hline & $\begin{array}{l}47-48-\text { Techniciens, contremaîtres } \\
\text { et agents de maîtrise }\end{array}$ & 4,017 & 7,5 & 6 \\
\hline & $\begin{array}{l}51-\text { Employés de la fonction } \\
\text { publique }\end{array}$ & 4,169 & 5 & 6 \\
\hline & $\begin{array}{l}54-\text { Employés administratifs } \\
\text { d'entreprise }\end{array}$ & 1,935 & 1 & 1 \\
\hline & 55 - Employés de commerce & 2,047 & 2 & 1 \\
\hline & $\begin{array}{l}56 \text { - Personnels des services directs } \\
\text { aux particuliers }\end{array}$ & 10,806 & 12 & 11 \\
\hline & $6-$ Ouvriers & Réf. & 4 & 11 \\
\hline \multirow[t]{5}{*}{$\begin{array}{l}\text { Tranche } \\
\text { d'effectifs de } \\
\text { l'établissement }\end{array}$} & Moins de vingt salariés & Réf. & 8 & 16 \\
\hline & De 20 à 49 salariés & 0,826 & 8 & 24 \\
\hline & De 50 à 499 salariés & 0,684 & 8 & 30 \\
\hline & Plus de 500 salariés & 0,741 & 9 & 22 \\
\hline & Effectifs inconnus ou non déclarés & 2,016 & 15 & 9 \\
\hline
\end{tabular}

Source: enquête «Emploi» 2005. Champs: ensemble des salariés.

Toutes les variables sont significatives au seuil de $1 \%$ sauf* $(5 \%)$.

Lecture: toutes choses égales par ailleurs, la probabilité d'effectuer de longues semaines est plus faible pour une femme que pour un homme. $5 \%$ des femmes effectuent de longues semaines. Les femmes représentent $27 \%$ des salariés effectuant de longs horaires. 
Cette première analyse statistique confirme la vision «habituelle» des salariés effectuant de longues durées de travail: le profil qui se dégage est conforme aux études effectuées dans d'autres pays développés et notamment aux États-Unis (SCHOR, 1991, JACOBS, Gerson, 1998 par exemple). Les TLD sont plus de deux fois plus fréquentes chez les hommes et cette association est visible également dans la régression logistique neutralisant l'effet des professions notamment (odd ratio (3) de presque 3,5 ). Le poids des contraintes domestiques et le partage traditionnel des tâches se traduit en partie dans cette situation. Cependant, il convient de nuancer cette vision exclusivement masculine de la population TLD en soulignant qu'il s'agit de tendances, parfois fortes, qui ne doivent pas faire oublier l'existence de salariés s'écartant de ce modèle. Ainsi les femmes représentent encore $27 \%$ des salariés TLD.

De même, le fait d'être en couple est davantage associé aux très longs horaires surtout si le conjoint ne travaille pas (odd ratio de 1,46) ce qui confirme à nouveau la possibilité d'une logique de spécialisation au sein du couple. Pour autant près des deux tiers des salariés TLD sont dans un ménage de deux actifs. Le lien entre longues durées et catégories professionnelles du conjoint a également été testé mais les résultats n'étaient pas significatifs. Par contre il semble bien que l'offre de travail des conjoints, lorsqu'ils sont tous deux actifs, soient davantage complémentaire que substituable (FERMANIAN, LAGARDE, 1998; BunEL, 2004). Les relations entre TLD et durée de travail du conjoint méritent néanmoins des recherches complémentaires.

Enfin, les tranches d'âge plus élevées sont associées à une plus grande probabilité d'effectuer des temps de travail très longs (odd ratio maximal pour les 40-50 ans). Cet effet, cohérent avec le constat d'une corrélation entre durée moyenne de travail et âge (BEFFy, 2006), reste néanmoins difficile à interpréter: il peut en effet être lié à l'âge mais également à un facteur générationnel (les salariés plus âgés conservant de longues durées) ou encore s'expliquer par le fait que les personnes travaillant de longues durées travaillent également à des âges plus élevés. Pour tenter d'éclaircir en partie cette question, nous pouvons d'une part remarquer que l'âge est lui-même corrélé à d'autres facteurs favorisant les longs horaires (encadrement, responsabilités, etc.). Cependant les traitements statistiques effectués sur le supplément «Conditions de travail» de l'enquête «Emploi» 2005 laissaient apparaître un effet d'âge y compris lorsque l'in-

(3) Les odds ratio ou «rapport des cotes»permettent de comparer l'impact sur la variable dépendante de chacune des modalités par rapport à celui de la modalité de référence (égale à 1). Ici il s'agit de mesurer l'impact du sexe sur la probabilité de connaître des horaires de 48 heures et plus. fluence des responsabilités hiérarchiques était écartée. D'autre part, l'étude des moyennes d'âges par groupes professionnels détaillés soulignent qu'au sein de chacun d'entre eux, la moyenne d'âge est plus élevée pour les salariés effectuant de longues durées, à l'exception des salariés du secteur public.

Ni le statut de l'employeur (public ou privé), ni le type de contrat (CDI, CDD, intérim...), ne modifient véritablement la fréquence des longues durées. En revanche les effectifs de l'établissement jouent un rôle modéré. Les longues durées sont ainsi un peu plus fréquentes au sein des petites structures.

L'élément qui ressort cependant le plus massivement est le poids des logiques professionnelles: le poids des cadres parmi les TLD demeure prédominant mais ces derniers sont accompagnés des professions intermédiaires d'entreprises ainsi que des employés des services aux particuliers. Si toutes les catégories socioprofessionnelles sont concernées, seules quelques professions, notamment lorsque l'on écarte le groupe cadre, concentrent l'essentiel des salariés TLD. Au sein des professions intermédiaires il s'agit principalement des professions intermédiaires commerciales (chef de vente, responsable de petite surface de vente, techniciens commerciaux et représentants, etc.). Mais cette concentration sur quelques professions est encore plus forte parmi les employés et ouvriers: ainsi les emplois familiaux (assistants maternels et aides à domicile), les serveurs, les gendarmes et pompiers et les chauffeurs constituent l'essentiel des salariés TLD. Effectuer des horaires très longs semble ainsi très dépendant de la profession. À côté du rôle de la profession, les corrélations avec les autres variables sociodémographiques (sexe, âge, situation familiale) ou professionnelles (contrat, employeur, effectifs de l'entreprise, etc.) sont bien plus faibles.

$\mathrm{Au}$ final, trois groupes professionnels principaux peuvent être distingués: les cadres et professions intermédiaires du privé (notamment dans les fonctions commerciales) regroupent environ la moitié des salariés concernés par les TLD, les employés et ouvriers occupant des métiers non ou peu qualifiés (environ un quart des longues durées) et enfin les salariés du secteur public (un quart également). Cependant la régulation du temps de travail de ces derniers obéit à des logiques spécifiques liées au principe de continuité du service public, c'est pourquoi nous ne l'étudierons pas ici pour nous concentrer sur les salariés du secteur privé (pour plus de détails sur les salariés du public et les contraintes temporelles qu'ils subissent voir DeVetter, 2004). 
Les temps longs des cadres et professions intermédiaires d'entreprise: un arbitrage en faveur du revenu et de la carrière?

La première grande catégorie concernée par les très longues semaines est la mieux connue: il s'agit des cadres et des professions intermédiaires d'entreprises (4). Des monographies ont été réalisées sur des salariés appartenant à cette catégorie (LEGAUT, Chasserio, 2006 par exemple) mais il n'y a pas, à notre connaissance, d'analyse plus générale sur les temps de travail particulièrement longs. Trois questions se dégagent de l'étude de ce premier groupe: peut-on isoler des métiers particulièrement concernés? Ces longues durées apportent-elles des contreparties élevées et peut-on dégager des explications à cette offre de travail «excessif»?

\section{Un élément de l’identité "cadre" mais inégalement réparti}

Bien que présentes pour l'ensemble des cadres et professions intermédiaires d'entreprises, les longues semaines touchent certains métiers de manière plus frappante encore (environ la moitié des cadres de la grande distribution, de l'hôtellerie ou des industries de métaux par exemple). Dans ces métiers, les longues durées de travail ne peuvent être considérées comme «exceptionnelles». Au contraire, non seulement elles apparaissent comme fréquentes mais aussi comme légitimes. Cette légitimité se diffuse par ailleurs à l'ensemble de la catégorie cadre pour laquelle le principe de durées supérieures à celles des autres salariés est largement admis. Les cadres (à temps plein) travaillent ainsi en moyenne 1870 heures par an contre 1650 pour l'ensemble des salariés à temps complet (BEFFY, 2006). Plus encore, les discours dominants insistent sur l'importance des temps de travail des cadres et ces durées peuvent être en partie considérées comme structurant l'identité de ces professions (KARVAR, RouBAn, 2004; Bouffartigue, 2004). Enfin des dispositifs juridiques spécifiques comme le forfait heures ou le forfait jours, mais également les spécificités des accords relatifs aux 35 heures pour les cadres appuient cette particularité est légitime en partie les dépassements d'horaires (LE GofF, 2003).

Cependant, si la plupart des professions appartenant à ces catégories sont concernées, l'observation des métiers les plus touchés (cadres commerciaux, techniciens commerciaux, ingénieurs d'études, chef de vente et maîtrise de l'hôtellerie par exemple) montre que les longues durées ne sont pas équita-

(4) Ce groupe correspond aux PCS 36 (cadres d'entreprise), 46 (professions intermédiaires administratives et commerciales des entreprises), 47 (techniciens) et 48 (contremaîtres et agents de maîtrise). blement réparties et souligne l'influence de deux facteurs principaux. Le premier est lié aux fonctions occupées: si en moyenne $18 \%$ des salariés de cette catégorie sont touchés, cette part atteint plus de $21 \%$ pour les fonctions de recherche et d'étude et surtout à plus de $27 \%$ pour les fonctions commerciales. Le second facteur est lié au rôle d'encadrement. L'impact des responsabilités hiérarchiques sur le temps de travail a été noté dans différentes études (Gershuny, 2005; Eastman, 1998). L'enquête «Emploi» ne permet pas de valider aisément cette hypothèse, mais il est possible, pour la tester, de recourir au supplément "Conditions de travail» 2005. Dans cette enquête les cadres et professions intermédiaires d'entreprises étaient $17 \%$ à travailler plus de 48 heures par semaine mais cette proportion grimpait à $25 \%$ lorsqu'ils avaient des salariés sous leurs ordres contre $9 \%$ dans le cas contraire. Cette concentration sur certaines professions ou fonctions rejaillit sur les caractéristiques sociodémographiques des salariés: les hommes constituent près de $85 \%$ des cadres TLD (contre $65 \%$ des autres cadres). Les TLD sont également davantage corrélées au fait d'être en couple (et plus encore à l'inactivité de l'épouse) que pour les autres catégories TLD. Cet élément renvoie à une vision beckerienne de l'allocation des temps et de la spécialisation des tâches. En ce sens, les durées supérieures à 48 heures hebdomadaires peuvent être considérées comme, en partie, choisies par les ménages, sous la contrainte des espérances salariales des conjoints. Mais ce choix s'effectue le plus souvent aux dépends de l'activité féminine (Legaut, Chasserio, 2006; Méda, Périvier, 2007; Petit, 2007). Limiter les durées maximales de travail est alors un élément essentiel d'une politique d'égalité des genres.

\section{Des durées payantes?}

La fréquence des longues durées pour certaines professions pose la question des contreparties obtenues en échange de cet investissement temporel très élevé. Pour cette première catégorie il semble que les contreparties soient relativement grandes. Elles concernent la rémunération et les perspectives de promotion, mais également des gains en termes d'autonomie et de maîtrise du temps de travail.

Les cadres disposent globalement de rémunérations élevées, mais plus encore ceux qui effectuent de longues durées. Ainsi pour cette première catégorie de salariés, la rémunération nette mensuelle moyenne est de 2050 euros pour les non TLD et passe à 3200 euros pour ceux qui effectuent de longues durées de travail, selon l'enquête «Emploi» 2005. Encore une fois, il est délicat d'établir le sens de la liaison rémunérations élevées - durées élevées: les temps longs peuvent permettre d'obtenir des salaires importants (heures supplémentaires, promotion liée à un investissement temporel important) ou à l'inverse les salariés recevant les rémunérations les plus fortes peuvent offrir des heures supplémentaires 
plus facilement dans une logique de don/contre-don ou de justification symbolique de la rémunération. De même, il est probable que la durée et la rémunération soient liées à des facteurs communs (encadrement par exemple). Néanmoins il y a une association claire entre rémunération élevée et longues durées. Cet avantage financier «statique» n'est pas le seul à être associé aux «durées excessives». De nombreux travaux (EASTMAN, 1998; KoDZ, 2003) soulignent sans ambiguiité le lien entre investissement temporel et perspectives de carrières. Le "présentéisme» (Silvera, 1998; Sheridan, 2004) est un facteur de promotion très répandu dans l'entreprise et cela d'autant plus que la productivité individuelle est inobservable comme c'est souvent le cas pour les fonctions d'encadrement et plus généralement dans de nombreuses activités de services. La mise en concurrence des salariés et leur évaluation sur la base du temps de présence (VAN ECHTELT et al., 2006; LANDERS et al., 1996; BRETt, STROH, 2003) peuvent expliquer le maintien de longues durées de travail parmi les cadres d'entreprise: le désir de travailler au moins autant que ses collègues peut éloigner durablement les durées offertes des durées souhaitées initialement. De même des mécanismes sociaux plus complexes sont également à l'œuvre à travers les pressions du marketing (Fraser, Paton, 2003; GeOrges, 2001) ou, dans une perspective plus sociologique, le désir de fournir «un travail ostentatoire» (GERSHUNY, 2005; Bowles, Park, 2001; Haight, 1997).

Parallèlement aux gains matériels, les longues durées de travail semblent ouvrir d'autres avantages à ceux qui les offrent. En effet, les longues durées sont associées à une autonomie et une maîtrise du temps de travail beaucoup plus grandes. Pour cette première catégorie de salariés TLD, le temps de travail est certes très long mais ceux-ci disposent d'une certaine maitrise de leurs horaires (et cela d'autant plus qu'ils travaillent longtemps): peu de contrôle, de nombreux arrangements possibles. Ici encore, l'enquête «Emploi» ne fournit que peu d'indications et il convient de recourir aux données du supplément «Conditions de travail» 2005: près de $56 \%$ des cadres et professions intermédiaires effectuant des semaines supérieures à 48 heures contre $20 \%$ de leurs homologues non TLD pouvaient déterminer librement leurs horaires (voir tableau 3 ). Plus encore, les cadres et professions intermédiaires TLD sont relativement épargnés par les heures «asociales » comme le travail du samedi, du dimanche ou de la nuit (relativement aux autres catégories de salariés travaillant longtemps). Même le travail le soir reste modéré par rapport aux autres catégories de salariés TLD (voir tableaux 3 et 4).

Ces différents gains obtenus par des durées très longues peuvent ainsi expliquer partiellement ce type d'offre de travail. Pour autant les différentes enquêtes menées (principalement au niveau européen) sur les préférences en termes de temps de travail soulignent le rejet très large des horaires
Tableau 3 : Salaires et temps de travail des cadres

\begin{tabular}{|l|c|c|}
\hline & $\begin{array}{c}\text { Cadres et PI } \\
\text { d'entreprises } \\
\text { non TLD }\end{array}$ & $\begin{array}{c}\text { Cadres et } \\
\text { PI d'en- } \\
\text { treprises } \\
\text { TLD }\end{array}$ \\
\hline Pourcentage de femmes & $35 \%$ & $15 \%$ \\
\hline Moyenne d'âge & 39 ans & 41 ans \\
\hline $\begin{array}{l}\text { \%o de salariés pouvant déter- } \\
\text { miner leurs horaires librement } *\end{array}$ & $20 \%$ & $56 \%$ \\
\hline Salaire mensuel ** & 2050 & 3200 \\
\hline $\begin{array}{l}\text { Travail habituellement } \\
\text { le dimanche }\end{array}$ & $4 \%$ & $6 \%$ \\
\hline Travail habituellement le samedi & $13 \%$ & $20 \%$ \\
\hline Travail régulièrement le soir & $7 \%$ & $18 \%$ \\
\hline $\begin{array}{l}\text { Travail régulièrement ou occa- } \\
\text { sionnellement de nuit }\end{array}$ & $12 \%$ & $15 \%$ \\
\hline
\end{tabular}

* Enquête «Conditions de travail» 2005.

** L'enquête «Emploi» n'est pas la meilleure source pour évaluer les salaires, mais les données fournies ici indiquent une tendance suffisamment claire pour soutenir le raisonnement.

Source: enquête «Emploi» 2005.

longs. Ainsi $81 \%$ de l'ensemble des salariés européens (5) travaillant 50 heures ou plus par semaine souhaitent diminuer leur temps de travail d'au moins 5 heures $(43 \%$ pour les salariés travaillant entre 40 et 49 heures) tandis que seuls $18 \%$ désirent conserver les mêmes horaires et $1 \%$ les augmenter (FAGAN et al., 2001). Ainsi même dans le cas des cadres où les TLD sont partiellement choisies il convient de bien relativiser la réalité de ce choix. Celui-ci reste largement sous contrainte (du revenu, de la pression des collègues, de la pression sociale, etc.). Plus encore l'arbitrage individuel éventuel est aussi perturbé par des contraintes organisationnelles fortes (manque de temps, responsabilité et encadrement, fonctions commerciales, contacts directs avec les clients, etc.) parfois instrumentalisées pour favoriser les longues durées de travail (UGHETTO, 2006). Les nouvelles méthodes de production ou de management qui tendent à se renforcer (comme le management par objectifs par exemple) conduisent ainsi à une intensification forte du travail (ASKEHNAZY, 2005; ARnAUdo et al., 2005). Face à celles-ci, certains salariés peuvent tenter de mobiliser davantage de temps professionnels. Le «choix » apparaît alors largement contraint. On rejoint ici les travaux de Coutrot (1998) sur le développement d'une forme «d'autonomie contrôlée» associant une relative autonomie des salariés à des contraintes organisationnelles fortes (délais à respecter, normes ISO, procédures techniques appuyées sur les technologies de l'information, etc.).

(5) Nous ne disposons malheureusement pas de données pour les seuls cadres, mais des enquêtes plus réduites appliquées à certaines professions cadres confirment le constat, voir par exemple EASTMAn, 1998. 
Les longues durées des employés et ouvriers: accepter des contraintes temporelles lourdes pour éviter un revenu salarial trop faible?

La visibilité de la première catégorie ne doit pas faire oublier la seconde, constituée d'employés et d'ouvriers (6). Certes les temps longs touchent une part plus faible de ces professions, mais ils se concentrent dans un nombre réduit d'activités: les transports (plus de $50 \%$ des marins, environ $25 \%$ des routiers), l'hôtellerie-restauration et surtout les emplois familiaux (assistants maternels, aides à domicile). Ainsi un tiers des assistants maternels travaillent plus de 48 heures par semaine. Les contreparties offertes aux longues durées de ce second type sont radicalement différentes du premier cas étudié. L'offre de ces temps de travail obéit ainsi à une toute autre logique.

\section{Des longues durées mais des salaires faibles}

Contrairement à ce que nous avions pu observer pour les cadres, les employés et ouvriers effectuant de longues durées ne sont pas parmi les mieux rémunérés de leur catégorie sociale. Ainsi, les rémunérations mensuelles des employés TLD sont proches de celles des employés travaillant des durées à temps plein inférieures à 48 heures (1380 euros contre 1160 euros mensuels). Ce constat peut signifier deux choses: soit les heures supplémentaires ne sont pas payées, soit elles sont effectuées par les salariés bénéficiant des salaires horaires les plus faibles. Cette seconde explication peut être en partie confirmée par la surreprésentation des assistants maternels dans les TLD. Ainsi il semble que les employés effectuant de longues durées soient concentrés dans les professions où le revenu habituel est le plus faible, effectuer des longs temps de travail serait alors un moyen de lutter contre la pauvreté.

La faiblesse des contreparties monétaires est renforcée, dans bien des cas, par l'absence de perspectives de carrière. En effet, les métiers les plus touchés se caractérisent par un niveau de formation initiale très faible et surtout par l'absence de marchés internes aux entreprises ou à la profession. L'écart entre le niveau inférieur et le niveau supérieur des grilles salariales est très faibles dans les professions touchées par les longues durées (absence de progression pour les assistants maternels, 6\% pour les organismes d'aide à domicile, $12 \%$ dans

(6) La catégorie étudiée regroupe les PCS 54 (employés administratifs d'entreprise), 55 (employés de commerce), 56 (personnels des services directs aux particuliers), 61 (ouvriers qualifiés), 66 (ouvriers non qualifiés) et 69 (ouvriers agricoles). l'hôtellerie-restauration). L'absence d'encadrement intermédiaire et le fait que les salariés TLD soient souvent plutôt âgés (typiquement pour les assistants maternels) limitent les opportunités de progression. Le poids des très petites entreprises et de l'emploi direct par les particuliers vont aussi dans ce sens. Un temps de présence long ne peut garantir une progression qui semble impossible. Cette situation est typique des métiers féminins qui représentent ici une part importante des employés TLD.

À l'absence de contreparties monétaires ou en termes de carrière, s'ajoute le fait que cette offre de durées très longues s'accompagne, contrairement à la situation des cadres, de contraintes temporelles très lourdes: travail le week-end fréquent, travail le soir ou la nuit, maîtrise très faible des temps de travail (et des RTT notamment), etc. Certes, les employés et ouvriers TLD subissent moins souvent des contrôles sur leurs horaires $(71 \%$ ne connaissent aucun contrôle contre $43 \%$ des employés et ouvriers non TLD selon l'enquête «Conditions de travail» 2005). Mais cette absence de contrôle ne se traduit que par un faible gain d'autonomie: ainsi plus des trois-quarts des salariés TLD de cette catégorie ne disposent pas de marges de manœuvres sur la détermination de leurs heures de travail.

Tableau 4 : Salaires et temps de travail des employés et ouvriers

\begin{tabular}{|l|c|c|}
\hline & $\begin{array}{c}\text { Employés et } \\
\text { ouvriers non } \\
\text { TLD }\end{array}$ & $\begin{array}{c}\text { Employés et } \\
\text { ouvriers TLD }\end{array}$ \\
\hline Pourcentage de femmes & $47 \%$ & $48 \%$ \\
\hline Moyenne d'âge & 38 ans & 41 ans \\
\hline $\begin{array}{l}\text { \% de salariés pouvant } \\
\text { déterminer leurs horaires } \\
\text { librement }\end{array}$ & $6 \%$ & $25 \%$ \\
\hline Salaire mensuel & 1160 euros & 1380 euros \\
\hline $\begin{array}{l}\text { Travail habituellement } \\
\text { le dimanche }\end{array}$ & $27 \%$ & $28 \%$ \\
\hline $\begin{array}{l}\text { Travail habituellement le } \\
\text { samedi }\end{array}$ & $14 \%$ & $49 \%$ \\
\hline $\begin{array}{l}\text { Travail régulièrement } \\
\text { le soir }\end{array}$ & $13 \%$ & $31 \%$ \\
\hline $\begin{array}{l}\text { Travail régulièrement } \\
\text { ou occasionnellement } \\
\text { de nuit }\end{array}$ & & $41 \%$ \\
\hline
\end{tabular}

Source: enquête «Emploi» 2005 sauf * enquête «Conditions de travail» 2005

\section{Des longues durées associées à une position dégradée sur le marché du travail}

La caractéristique centrale de cette seconde catégorie de salariés TLD est la position très dégradée qu'ils occupent face à l'employeur. Ils appartien- 
nent au segment secondaire du marché du travail et cumulent en effet un certain nombre d'effets négatifs sur ce plan: les secteurs concernés sont peu syndiqués et la part des salariés directement employés par un particulier ou par une très petite entreprise est forte (près des deux tiers appartiennent ainsi à des établissements de moins de cinquante salariés). Les secteurs les plus touchés sont connus pour les libertés que certains employeurs prennent avec le Code du travail (restauration, transports, $c f$. Syratef-CFDT, 1997) tandis que d'autres échappent au contrôle de l'inspection du travail du fait de l'exercice de la profession au domicile du salarié (assistant maternel) ou de l'employeur (aide à domicile, employé de maison). Parallèlement les salariés occupent une position fragile sur le marché du travail notamment parce qu'ils sont très peu diplômés : $35 \%$ n'ont aucun diplôme et seuls $18 \%$ ont un diplôme niveau bac ou supérieur (contre plus de $23 \%$ des ouvriers et employés non TLD).

Enfin plusieurs de ces métiers sont marqués par la difficulté d'y réaliser des gains de productivité: le travail consistant en des tâches de surveillance (assistant maternel), d'entretien ou (partiellement) d'attente (hôtellerie-restauration, chauffeurs). Cette porosité du temps de travail a conduit à des conflits de délimitation du travail effectif comme l'illustrent les négociations dans l'hôtellerie-restauration ou les transports routiers. La notion d'heures d'équivalence souligne la faible reconnaissance des heures de travail effectuées dans certains cas. En effet, au sein d'un certain nombre d'emplois pour lesquels existent des périodes d'inactivité ou de moindre activité, des équivalences entre durée de travail effectuée et durée comptabilisée ont été mises en place. Ces périodes d'équivalence ne constituent pas alors des temps de travail effectifs mais sont «rémunérés conformément aux usages et conventions ou accords collectifs » (article L. 212-4 du Code du travail). Ce mécanisme tend plutôt à décroître mais concerne encore un certain nombre de professions le plus souvent considérées comme peu qualifiées (les bateliers, les gardiens d'immeubles, les gardiens de chantiers ou encore les fonctions peu qualifiées des hôpitaux et cliniques, etc.). Il convient cependant de remarquer que la jurisprudence de la Cour de justice des Communautés européennes tend à considérer les heures d'équivalence comme du travail effectif entrant ainsi dans le calcul d'une durée hebdomadaire ne pouvant légalement dépasser 48 heures (cet arrêt de 2005 ne modifie cependant pas les modalités de calcul de la rémunération).

La situation défavorable des salariés face aux employeurs conduit à apporter des explications très différentes à l'offre de temps de travail très longs des employés et ouvriers par rapport aux logiques dominantes chez les cadres. Les contraintes qui pèsent sur les offreurs au moment de l'arbitrage entre-temps libre et revenu sont particulièrement fortes et parler de choix peut apparaître très délicat.
Les rares travaux sur les longues durées qui ne soient pas focalisés sur les salariés qualifiés soulignent plutôt la dépendance de ces horaires vis-à-vis de la demande de travail et il semble que l'élément déterminant soit le rapport de force entre salariés et employeurs. Les longues durées de travail se retrouvent principalement dans les pays où le marché du travail est peu régulé (O'ReILly, 2003). Elles concernent davantage les travailleurs non syndiqués et sont fortement alimentées par l'accroissement des heures supplémentaires non payées (particulièrement nombreuses au Royaume-Uni ou en Australie). SCHOR $(1991,2005)$ insiste sur cet aspect: 1'accroissement de la durée du travail aux États-Unis durant les deux dernières décennies résulte d'abord de la détérioration de la position des salariés vis-à-vis des employeurs. Les études de cas sur les salariés effectuant de longs horaires menés sous la direction de Kodz montrent également que l'une des principales satisfactions liées aux temps de travail élevés provient du sentiment que cette pratique augmente les chances de conserver son emploi (Kodz, 2003, p. 217).

Le pouvoir patronal sur la détermination des horaires de travail joue ainsi un rôle important et cela d'autant plus facilement que les salaires horaires sont très faibles. Plus encore, dans plusieurs des métiers fortement touchés, le salaire est en partie «à la tâche» (assistants maternels, chauffeurs, serveurs, etc.). Dans ces conditions allonger le temps de travail permet d'accroître des rémunérations jugées insuffisantes. La contrainte financière complète (ou remplace) les pressions directes de l'employeur pour obtenir une durée élevée de travail comme pour obtenir une disponibilité ou une flexibilité élevée (DEVETTER, 2002).

Enfin, certaines formes d'organisations du travail peuvent favoriser une offre contrainte de longues durées. C'est le cas notamment du poids du rapport direct avec des clients ou des usagers (UGHETTO, 2006). Lorsque le bénéficiaire n'est pas un individu anonyme, mais un acteur avec qui l'employé noue des contacts personnels plus ou moins forts (c'est le cas des assistants maternels ou des aides à domicile et des professions de santé), le poids du devoir moral pousse à offrir une disponibilité élevée qui «ne peut être» rémunérée car elle n'a pas été demandée par l'employeur en tant que telle. La relation personnalisée avec le bénéficiaire du service dégrade encore le pouvoir de négociation des salariées et accroît l'exploitation potentielle (FolBRE, 1997; England, 1998; Jany-Catrice et al., 2004). Selon l'enquête «Conditions de travail» 2005, parmi les employés et ouvriers (hors fonction publique) ne travaillant pas plus de 48 heures par semaine, $59 \%$ sont en contact direct avec le public, mais c'est le cas de $77 \%$ de ceux qui connaissent des semaines très longues. De même les situations d'isolement et notamment l'absence de collègue sont liées à une plus grande fréquence de longues durées hebdoma- 
daires: seuls $37 \%$ des employés et ouvriers TLD peuvent se faire aider de leurs collègues contre $68 \%$ de leurs homologues travaillant moins longtemps. Le constat est similaire dans le cas de l'aide d'un supérieur hiérarchique.

Au final les métiers peu qualifiés touchés par les longues durées cumulent de nombreux désavantages. Le travail est peu valorisé car il apparaît lié à des tâches non nobles de surveillance, de conduite, d'attente mais pas de "production» matérielle visible. Ils ne mobilisent pas de compétences reconnues mais plutôt des capacités jugées naturelles (assistants maternels) ou largement partagées (chauffeurs). Les salariés sont souvent isolés par rapport à leurs collègues, mais sont au contraire au contact direct avec les clients ou les usagers. Ce sont enfin des emplois où la délimitation du travail «effectif» est conflictuel: certaines heures sont considérées comme ne valant pas 60 minutes de travail(7). Les durées conventionnelles de travail dépassent souvent la durée légale ( 45 heures hebdomadaires dans la convention collective des assistants maternels par exemple).

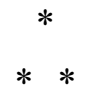

Les études visant à comprendre le maintien (voire le développement dans les pays anglosaxons) des longues durées de travail ont proposé de nombreuses explications. Certaines s'appuient sur l'offre de travail tandis que d'autres insistent sur le rôle de la demande et des pressions des employeurs. Derrière cette opposition se pose la question du choix des longues durées par les salariés : ces derniers offrent-ils des temps de travail très longs ou répondent-ils à une injonction de l'employeur? Cette question du choix est déterminante dans le cadre du débat européen sur la révision de la directive «temps de travail». En effet, les arguments en faveur du maintien de la clause d'optout reposent principalement sur l'importance du libre choix des travailleurs. Or ce choix n'est pas toujours évident à repérer. Ensuite, et même si le principe d'une demande de la part des salariés est retenu, il convient de comprendre les motivations à la base de cette demande (préférence pour le revenu, logique de "contagion sociale», «compétition par les heures», etc.). Les résultats des analyses précédentes sont ainsi souvent contradictoires.
Il est en effet délicat de privilégier une explication au détriment des autres, plusieurs situations pouvant coexister en s'appliquant spécifiquement à telle ou telle profession ou catégorie sociale. Dans le cas de la France, deux grandes logiques (en dehors de celle liée au service public) semblent se dégager: à une logique d'investissement professionnel important en partie choisi et permettant d'atteindre des positions relativement privilégiées s'opposent des longues durées bien moins maîtrisées et supportées par des professions bénéficiant de salaires horaires très faibles.

Les coûts sociaux, familiaux et médicaux touchent certes toutes les catégories, mais il n'en va pas de même pour les avantages reçus. Ainsi si les temps de travail excessifs d'une partie des cadres retiennent l'attention, ils ne doivent pas faire oublier la situation très dégradée de certaines professions employées ou ouvrières. Comme pour d'autres dimensions de la disponibilité temporelle (comme la prévisibilité des horaires) deux modèles s'opposent: la disponibilité de l'expert impliquant une forte pénibilité temporelle mais également des avantages et une reconnaissance sociale conséquents se distingue d'une disponibilité liée à la non-qualification et à la dépendance vis-à-vis des donneurs d'ordres (souvent employeurs). La faiblesse des rémunérations et l'absence de reconnaissance sociale s'ajoutent alors aux contraintes temporelles. Dans les deux cas cependant, une régulation publique semble nécessaire pour limiter des offres de travail excessives au regard des coûts sociaux qui y sont associés, comme le reconnaît notamment la charte des droits fondamentaux de l'Union européenne (de 2000) qui stipule que tout travailleur a droit à une limitation de la durée maximale du travail. 


\section{Bibliographie}

Arnaudo B. et al. (2005), «Exposition aux risques et aux pénibilités du travail de 1994 à 2003 : premiers résultats de l'enquête SUMER 2003 », Documents pour le médecin du travail, $\mathrm{n}^{\circ} 101$.

AskenAzy P. (2004), Les désordres du travail, enquête sur le nouveau productivisme, collection «La Républiques des Idées », Paris, Édition du Seuil, 95 p.

Beffy M. (2006), «Les salariés à temps complet travaillent 1650 heures dans l'année», INSEE Première, $\mathrm{n}^{\mathrm{o}} 1066$, février.

Bescond D., Châtaignier A., Mehran F. (2003), «Sept indicateurs pour mesurer le travail décent: une comparaison internationale», Revue internationale du travail, volume 142, no 12 , pp. 195-229.

Bouffartigue P. (2004), Le Retour des Classes Sociales, Paris, La Dispute.

Bourdieu J., Reynaud B. (2004), «Discipline d'atelier et externalités dans la réduction de la durée du travail au XIX $\mathrm{X}^{\mathrm{e}}$ siècle», pp. 14-53, in Fridenson P., Reynaud B. (sous la direction). La France et le temps de travail (1814-2004), Paris, éditions Odile Jacob.

Bowles S., Park Y. (2005), "Emulation, Inequality, and Work Hours: Was Thorsten Veblen Right?" Economic Journal, Vol. 115, No. 507, pp. F397-F412, November.

Brett J., Stroh L. (2003), "Working 61 plus hours a week: why do managers do it?", Journal of Applied Psychology, vol. 88, no 1, 67-78.

Bunel M. (2004), «Les conjoints des salariés passés à 35 heures travaillent-ils davantage?», Économie et Prévision, no $164-165$, pp. 165-188.

Coutrot T. (1998), L'entreprise néolibérale, nouvelle utopie capitaliste? collection «Textes à l'Appui», Paris, La Découverte.

Devetter F.-X. (2002), «Quelle convention post-fordiste de disponibilité temporelle au travail?», CEREQ, Formation Emploi, no 78, juin 2002.

Devetter F.-X. (2004), «Les employés du public: un régime temporel spécifique?», Politique et Management Public? No $1 / 2004$.

EASTMAn C. (1998), "Working for position: Women, Men and Managerial Work Hours", Industrial Relations, Vol. $37, \mathrm{n}^{\mathrm{o}} 1$, pp. 51-65.

ENGLAND P. et al. (2002), "The wage of virtue: relative pay of care work", Social Problems, 49: 455-473

FAGAN C. (2004), "Gender and working time in industrialized countries", in Working time and workers preferences in industrialized countries, Messenger Jon, Routledge.

Fermanian J.-D., Lagarde S. (1998), «Les horaires de travail dans le couple», Économie et Statistique, no 321 322, pp. 89-110.
Folbre N. (1995), "Holding Hands at midnight: the pradox of caring labor", Feminist Economics, Vol. 1, $\mathrm{n}^{\circ} 1$, Spring 1995.

Fraser S., Paton D. (2003), "Dœs Advertising increase labour supply? Time series evidence from the UK". Applied Economics, 35, 1357-1368.

Georges D. (2001), “Working Longer Hours: Pressure from the boss or Pressure from the Marketers? "Review of Social Economy, Vol. LV, no 1, Spring 197, pp. 33-63.

Gershuny J. (2005), "Busyness as the Badge of Honor for the New Superordinate Working Class. Social Research, Summer", Vol. 72 Issue 2, pp. 287-314.

Givord P. (2003), «Une nouvelle enquête emploi», Économie et Statistiques, no 362, pp. 59-67.

Godet M. (2006), «L'erreur collective des 35 heures», Le Monde, 7 février.

Haight A. (1997), "Padded Prowess: a Veblenian Interpretation of long hours of salaried workers", Journal of Economic Issues, Vol. XXXI, n ${ }^{\circ}$ 1, march, 29-38.

JACOBS J. , Gerson K. (1998) "Who are the overworked Americans?" Review of social Economy, Vol. LVI, no 4, Winter 1998, pp. 443-459.

Jany-Catrice F., Gadrey N., Pernod-Lemattre M. (2004), «En 2002, près des deux tiers des non qualifiés sont des employés», Premières Informations et Premières Synthèses, Dares, no 49-1, décembre.

Karvar A., Rouban L. (2004), Les cadres au travail, Paris, La Découverte.

Kodz J. et al. (2003), "Working Long hours: a review of the evidence", Vol. 1 and 2, Employment Relations Research Series, $\mathrm{n}^{\mathrm{o}} 16$.

Landers R., Rebitzer J., TAYlor L. (1996), "Rat race redux: Adverse selection in the determination of Work hours in Law firms", American Economic Review, Vol. $86, n^{\circ} 3$, pp. 329-348.

Le Goff, J. (2003), Droit du travail et société, Presses Universitaires de Rennes.

Legaut M.-J., Chasserio S. (2006), «L'organisation du travail par projets dans les services technologiques aux entreprises, longues heures et effets différenciés selon le genre», colloque travail emploi formation, Lille, 2324 novembre.

Méda D., Périvier H. (2007), Le Deuxième âge de l'émancipation, collection «La République des Idées», Paris, éditions du Seuil.

Missègue N. (2000), «Le temps de travail des indépendants », INSEE Première, $\mathrm{n}^{\circ}$ 695, janvier.

O’Reilly J. (ed) (2003), Regulating Work time Transitions in Europe, Edward Elgar Publishing, Nothampton. 